\title{
THE SHORTED ANNULAR PATCH AS A MULTI-PATH SUPPRESSING ANTENNA
}

\author{
G.J.K. Moernaut*, G.A.E Vandenbosch ${ }^{\dagger}$ \\ *Orban Microwave Products, Belgium, Gerald.Moernaut@orbanmicrowave.com \\ ${ }^{\dagger}$ KULeuven, Belgium, Guy.Vandenbosch@esat.kuleuven. be
}

Keywords: patch antenna, reduced surface wave, multi-path suppression, GNSS

\begin{abstract}
This paper presents an overview of the multi-path phenomena and the performance of the Shorted Annular Patch (SAP) in suppressing unwanted multi-path signals caused by ground reflections. It is shown that the SAP's previously derived multi-path suppressing optimum is in fact sub-optimal. An improved design rule based on the Multi-Path Ratio (MPR) is proposed.
\end{abstract}

\section{Introduction}

This paper discusses the multi-path caused by ground reflections and quantifies the antenna susceptibility to this multi-path, namely the Multi-Path Ratio (MPR). The basic topology of the Shorted Annular Patch (SAP) is briefly outlined. The original multi-path suppression optimum is given. The performance of the SAP antenna is studied making use of the MPR definition. An improved multi-path suppression optimum is defined based on the MPR definition. A theoretical link between the old and new multi-path suppression optimum is established.

\section{The multi-path problem in GNSS}

Multi-path is a prime source of navigation errors in GNSSbased navigation systems. Multi-path errors arise from interference between the received direct and reflected signals in the receiver. Multi-path mitigation algorithms have been developed and are embedded within the digital part of most high-end receivers. Since it is not possible to provide any multi-path suppression in the RF part of the receiver, the antenna remains the only possibility to provide additional multi-path suppression. Multi-path signals arrive mainly at low elevations, due to vertical obstacle reflections, and from the bottom, due to ground reflections. The antenna's susceptibility to multi-path signals is mainly due to its radiation properties at low elevations. This low elevation radiation can largely be subscribed to surface wave propagation on the ground plane. These surface waves are scattered at the ground plane edge, giving rise to unwanted radiation towards the back of the antenna and at low elevations. The antenna's multi-path performance is popularly quantified with the Multi-Path Ratio (MPR).
The MPR for ground reflections can be straightforwardly arrived at by inspecting figure 1 depicting the multi-path problem caused by ground reflections. The desired GNSS signals are Right Hand Circularly Polarized while the undesired ground reflected signals might be Left Hand Circular Polarized as well as RHCP.

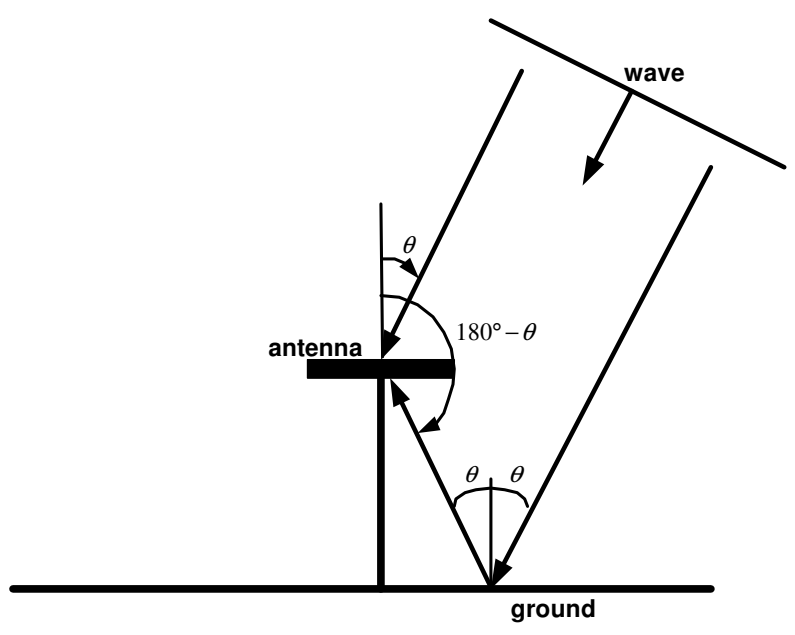

Figure 1: The multi-path problem quantified.

The multi-path susceptibility of a GNSS antenna can be quantified by the MPR defined as:

$$
M P R=\frac{E_{R H C P}(\theta)}{E_{R H C P}\left(180^{\circ}-\theta\right)+E_{L H C P}\left(180^{\circ}-\theta\right)}
$$

Where $\mathrm{E}_{\mathrm{RHCP}}$ and $\mathrm{E}_{\mathrm{LHCP}}$ are the antenna's directivity (or gain) at $\theta$ or $\theta$ 's supplement angle $180^{\circ}-\theta$.

Equation 1 clearly demonstrates the importance of two antenna characteristics, namely:

- The front-to-back ratio:

If there is no radiation sensitivity towards the ground, the denominator becomes zero and as a result the MPR becomes infinite. Hence, the antenna has perfect multi-path performance for ground reflections.

- The axial ratio: The MPR formula has an RHCP to LHCP ratio embedded within, which at $0^{\circ}$ elevation, or $\theta=90^{\circ}$ becomes an RHCP to LHCP ratio at the same $0^{\circ}$ 
elevation. This is popularly designated as the axial ratio of an antenna.

\section{The shorted annular patch}

The Shorted Annular Patch (SAP), whose topology is shown in figure 2, was originally proposed by D.R. Jackson et al. as a Reduced Surface Wave (RSW) patch antenna [2]. This patch antenna was shown not to excite surface waves when a certain RSW condition is met. Unwanted low elevation radiation is minimized when surface wave excitation is avoided. This leads to an increased gain roll-off towards lower elevations. This increased gain roll-off is the reason the SAP was given the multi-path suppression capable status [1].

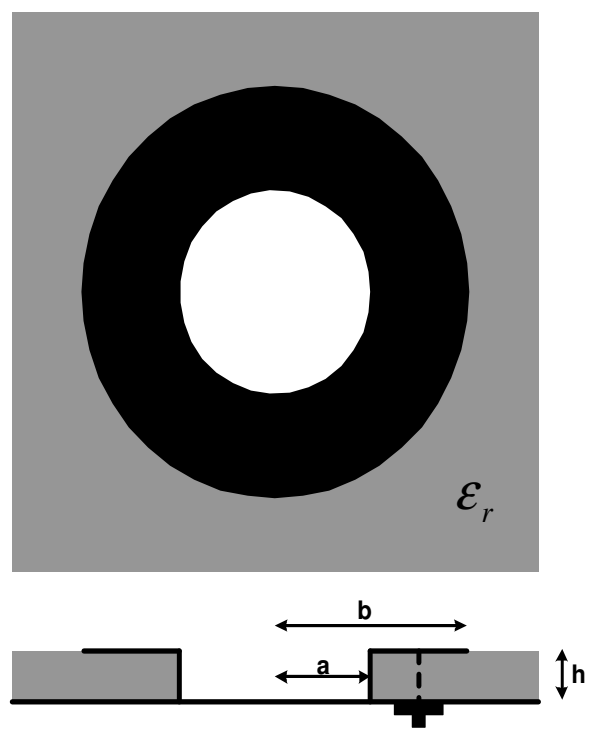

Figure 2: The SAP topology.

The resonance condition of an SAP antenna as derived using the classic cavity model and is given by $[2,3]$ :

$$
J_{n}{ }^{\prime}\left(\frac{b}{a} \mathrm{X}_{n m}\right) Y_{n}\left(\mathrm{X}_{n m}\right)-J_{n}\left(\mathrm{X}_{n m}\right) Y_{n}^{\prime}\left(\frac{b}{a} \mathrm{X}_{n m}\right)=0
$$

With,

$$
f_{n m}=\frac{\mathrm{X}_{n m} c}{2 \pi a \sqrt{\varepsilon_{r}}}
$$

The variables in equation 2 and 3 are:

- $\quad b$ and a are the outer and inner radius of the SAP.

- $\mathrm{X}_{\mathrm{nm}}$ is a root of equation 2 corresponding with mode $\mathrm{n}$ (radial) - $\mathrm{m}$ (axial).

- $\mathrm{J}_{\mathrm{n}}($.$) and \mathrm{Y}_{\mathrm{n}}($.$) are the Bessel functions of the first$ and second kind of order $n$.

- $\mathrm{J}_{\mathrm{n}}($.$) and \mathrm{Y}_{\mathrm{n}}{ }_{\mathrm{n}}($.$) are the derivates of the Bessel$ functions of the first and second kind of order $\mathrm{n}$ with respect to their argument.

- $\mathrm{f}_{\mathrm{nm}}$ is the resonance frequency of the nm-mode.
- $\quad \mathrm{c}$ is the velocity of light in free space.

- $\varepsilon_{r}$ is the dielectric constant of the SAP's substrate.

Calculating the resonance frequency of the SAP involves solving the transcendental equation 2 for which an iterative procedure is required. Another simplified way for calculating the SAP's resonance frequency avoiding an iterative procedure has been published in [3].

The original multi-path suppression condition, which is in fact the RSW condition, is [2]:

$$
\begin{aligned}
& J_{1}^{\prime}\left(\beta_{T M_{0}} b\right)=0 \\
& \beta_{T M_{0}} b=x_{1 m}=1.8412
\end{aligned}
$$

The variables in equation 4 and 5 are:

- $\quad \mathbf{J}^{\prime}{ }_{1}($.$) is the derivate of the Bessel functions of the$ first kind of order 1 with respect to its argument.

- $\beta_{T M_{0}}$ is the $\mathrm{TM}_{0}$ surface wave wave number. The $\mathrm{TM}_{0}$ surface wave is the only surface wave present in a thin low permittivity dielectric slab [4].

- $\chi_{\mathrm{nm}}$ is a root of equation 4 and is referred to as the RSW figure. The MPR condition remains described as in terms of RSW in order to keep the relation to its original conception.

Note that only the first solution of equation 4 is considered because this results in the smallest SAP outer radius and excites the SAP in its fundamental $\mathrm{TM}_{11}$ mode.

The surface wave root can be found using the procedure outlined in [4]. $\beta_{T M_{0}}$ can be approximated by $\mathrm{k}_{0}$, the free space wave number, for thin low permittivity dielectric slabs.

\section{Shortcomings of the original multi-path suppression condition}

This multi-path suppression condition is in fact a reduced surface wave condition. If the condition is met, the antenna does not excite surface waves. These surface waves give rise to radiation at low elevations and scattering at the substrateground plane truncation. This scattering results in backward radiation worsening the front-to-back ratio of the antenna. However, the front-to-back ratio is not the only important parameter. The axial ratio is very important as well; especially a good axial ratio at low elevations is desired. Unfortunately, blocking radiation at low elevation leads to degraded axial ratio performance since a minimum radiation is needed in order to ensure good circular polarization radiation.

In order to demonstrate the axial ratio degradation when the SAP's outer radius meets the original RSW optimum, an SAP on a RO4003 substrate from Rogers Corporation ${ }^{\mathrm{TM}}$ of 1.524 $\mathrm{mm}$ thickness was simulated with CST Microwave Studio ${ }^{\text {TM }}$. Fringing field compensation has been applied according to the procedure in [3]. The substrate and ground plane radii were 
taken $20 \mathrm{~mm}$ larger than the SAP's outer radius. This topology is referred to as TOPA. Figure 3 and 4 show a 2 dimenional cross section of the RHCP and LHCP radiation pattern for three RSW figures, namely 1.75 (-), 1.85 (x), which is close to 1.8412 being the original RSW optimum, and $1.95(\mathrm{o})$.

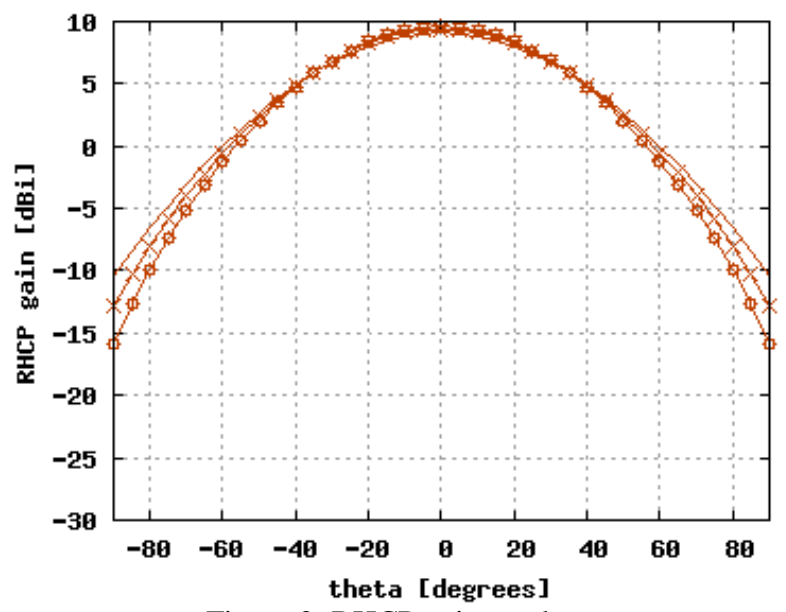

Figure 3: RHCP gain vs. theta.

The RHCP gain roll-off increases with increasing RSW figure as expected, while the LHCP gain is higher as well for increased RSW figures.

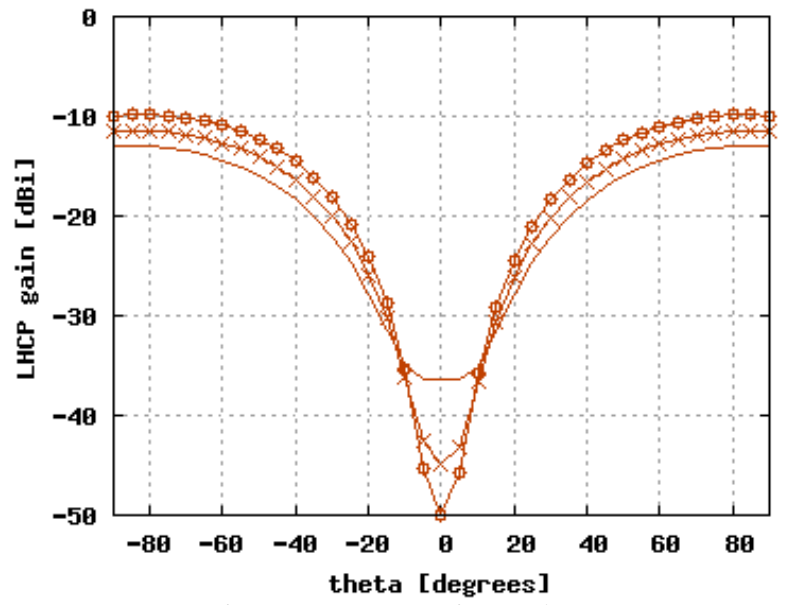

Figure 4: RHCP gain vs. theta.

Figure 5 displays the axial ratio for the same SAP implementations.

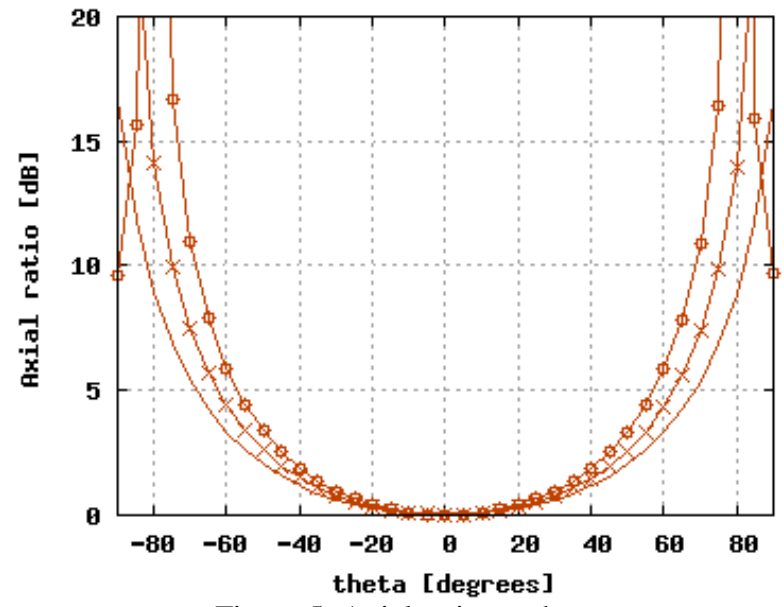

Figure 5: Axial ratio vs. theta.

Axial ratio degradation with increasing RSW figure is readily observed. Since the axial ratio has a profound influence on the multi-path performance, the MPR of the same SAP implementations is plotted in figure 6 .

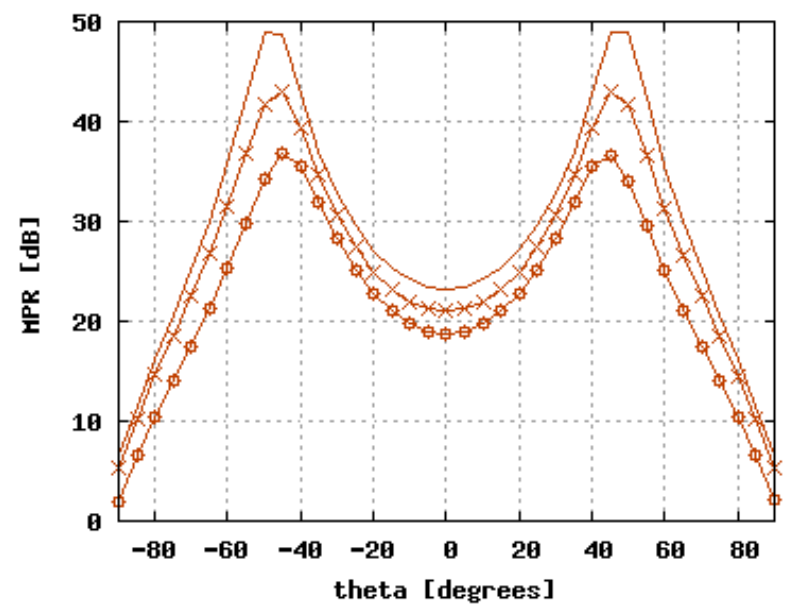

Figure 6: Multi-path ratio vs. theta.

Figure 6 clearly demonstrates the multi-path performance degradation with increasing RSW figure including the original RSW optimum. The MPR and axial ratio are seen to improve for decreasing RSW figure. However, since the radiation at low elevation increases with decreasing RSW figure. This leads to a lower gain roll-off implying a degraded front-to-back ratio, which is vital for proper multi-path performance as well. An MPR optimum RSW figure is expected from this observation.

\section{Improved multi-path suppression condition}

In order to find a multi-path optimum RSW figure the SAP was simulated over a wider range of RSW figures. The MPR was plotted as a function of the RSW figure for three elevations, namely $5^{\circ}(-), 10^{\circ}(\mathrm{x})$ and $15^{\circ}(\mathrm{o})$ in figure 7 . An MPR optimum around an RSW figure of 1.73 is observed. 


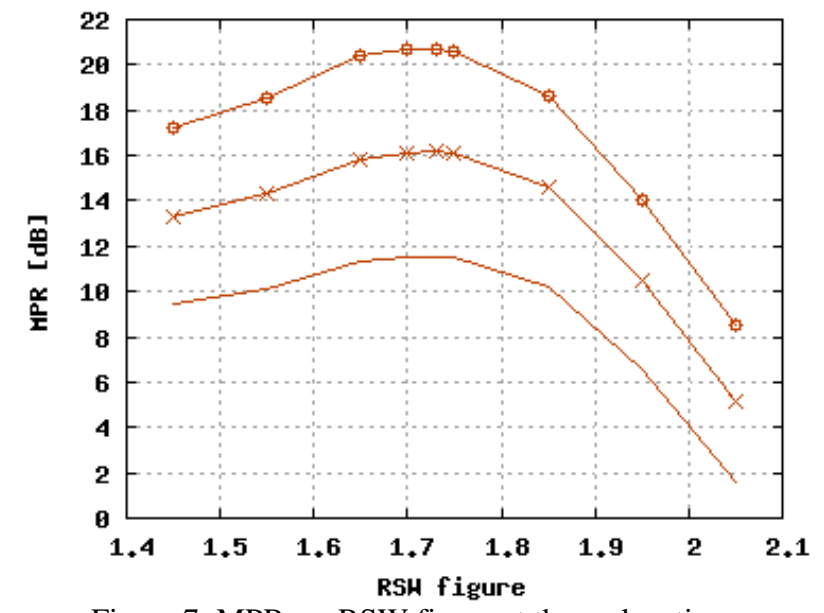

Figure 7: MPR vs. RSW figure at three elevations.

The RHCP gain and axial ratio for the same elevations are plotted as a function of the RSW figure in plots 8 and 9. These figures clearly demonstrate the common misunderstanding that a higher gain roll-off automatically yields a higher multi-path suppression, which is definitely not the case. The discussion clearly shows the axial ratio has an underestimated role in multi-path suppression.

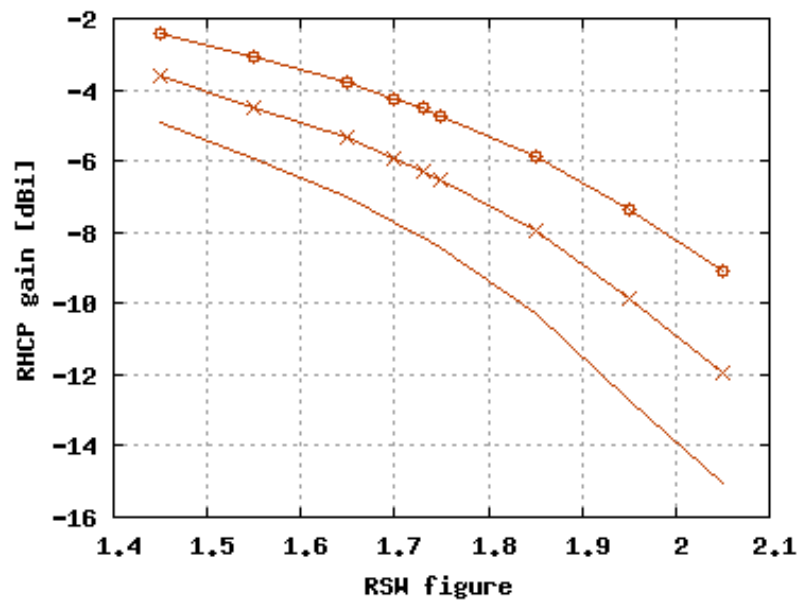

Figure 8: RHCP gain vs. RSW figure at three elevations.

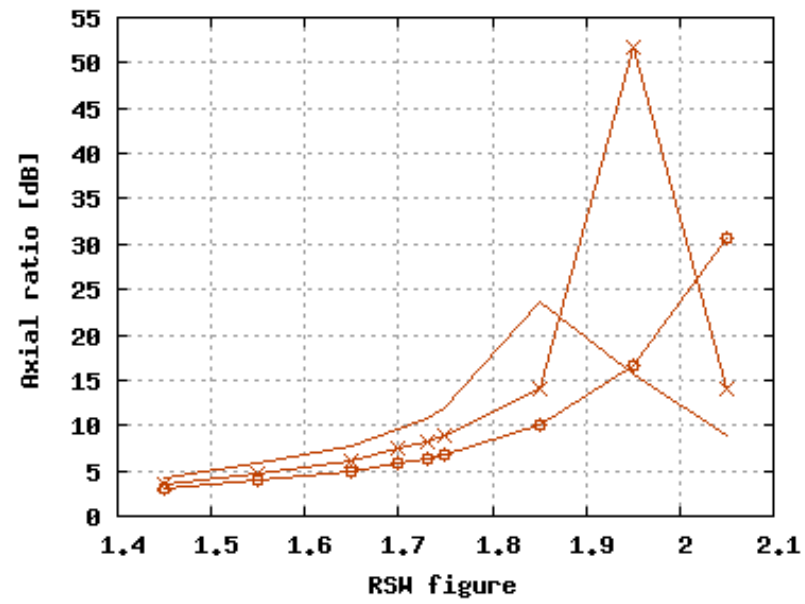

Figure 9: Axial ratio vs. RSW figure at three elevations.

A serious axial ratio degradation for larger RSW figures is observed in figure 9 accompanied with an increasing RHCP gain roll-off as seen in figure 8 .

In order to verify the generality of the new MPR optimum RSW figure, the SAP was simulated on a $3.25 \mathrm{~mm}$ thick RO4003 substrate with $20 \mathrm{~mm}$ ground plane extension (TOPB), a $1.524 \mathrm{~mm}$ thick RO4003 substrate with $40 \mathrm{~mm}$ ground plane extension (TOPC) and a $1.28 \mathrm{~mm}$ RO3006 substrate with $20 \mathrm{~mm}$ ground plane extension (TOPD). The discussion is limited to the SAP's first mode only. This limits the dielectric constant to 8.4, the critical dielectric constant [2]. The MPR as a function of the RSW figure for $5^{\circ}(-)$, $10^{\circ}(\mathrm{x})$ and $15^{\circ}(\mathrm{o})$ elevations is shown in figures 10 to 12 .

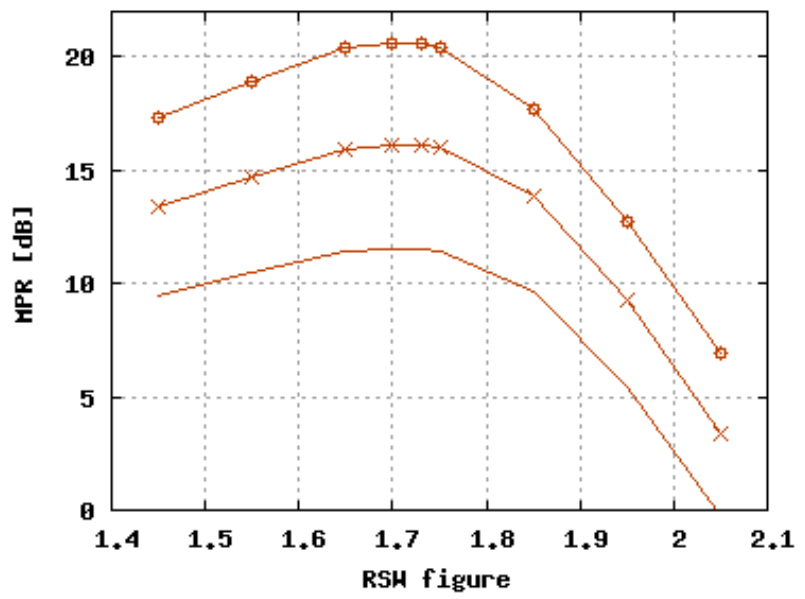

Figure 10: MPR vs. RSW figure at three elevations of TOPB. 


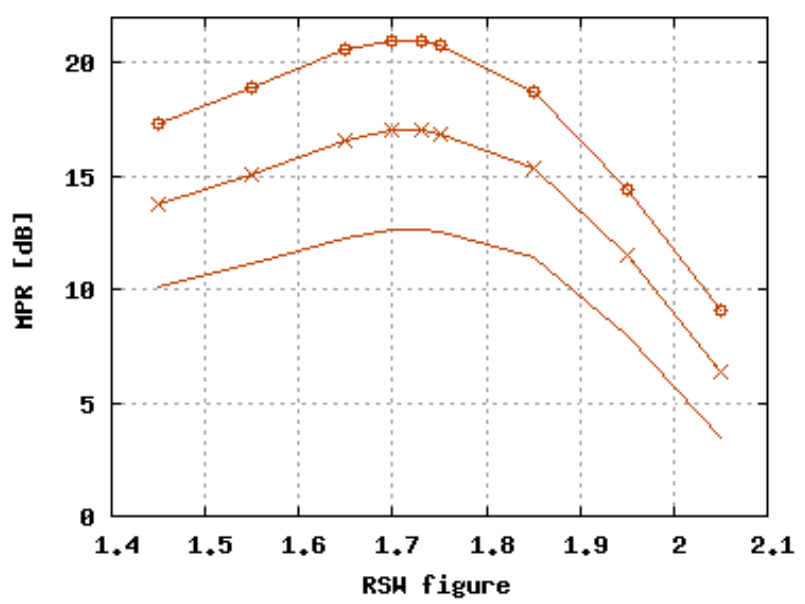

Figure 11: MPR vs. RSW figure at three elevations of TOPC.

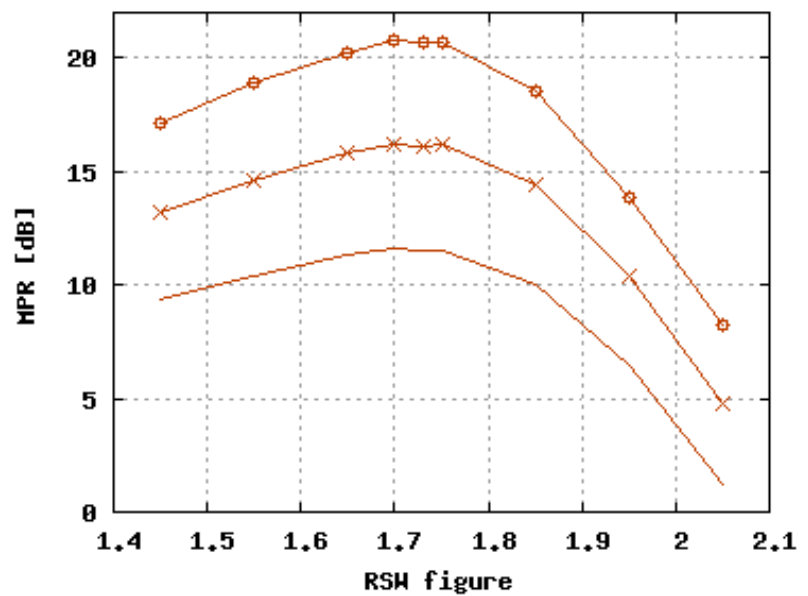

Figure 12: MPR vs. RSW figure at three elevations of TOPD.

Figure 10 to 12 clearly demonstrate the generality of the new optimum RSW figure equal to 1.73 . This new optimum agrees well with the intuitively expected, namely:

- The new MPR optimum value is lower than the original one. This results in increased radiation at lower elevations needed for good circular polarization radiation.

- The new MPR optimum remains close to the original value, which minimizes gain roll-off degradation. Hence, the front-to-back ratio of the new multi-path optimum remains within the vicinity of the old multi-path optimum.

\section{Link with the original RSW condition equation}

The original RSW definition as given in equation $4(-)$ is plotted as a function of the RSW in figure 13 and 14 .

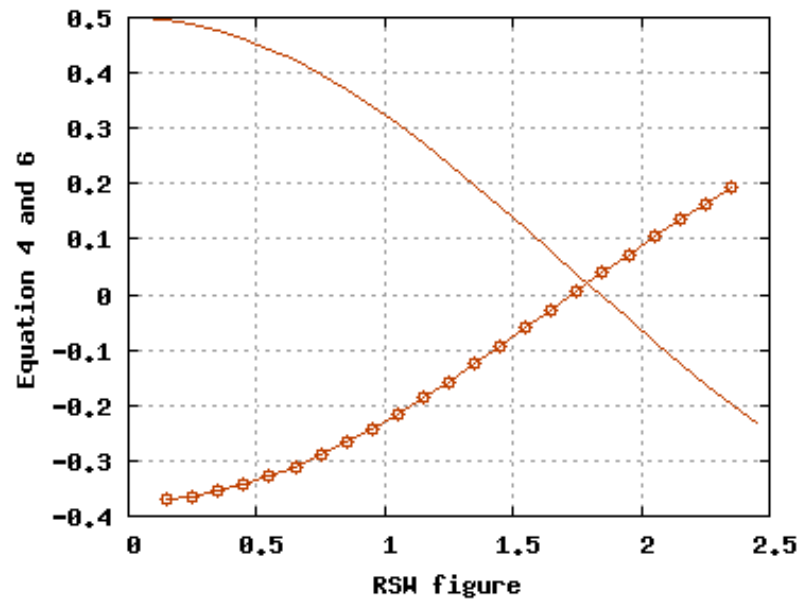

Figure 13: Equation 4 and 6 vs. RSW figure coarse.

Figure 13 is enlarged in the vicinity of both the old and the new optimum for clarity.

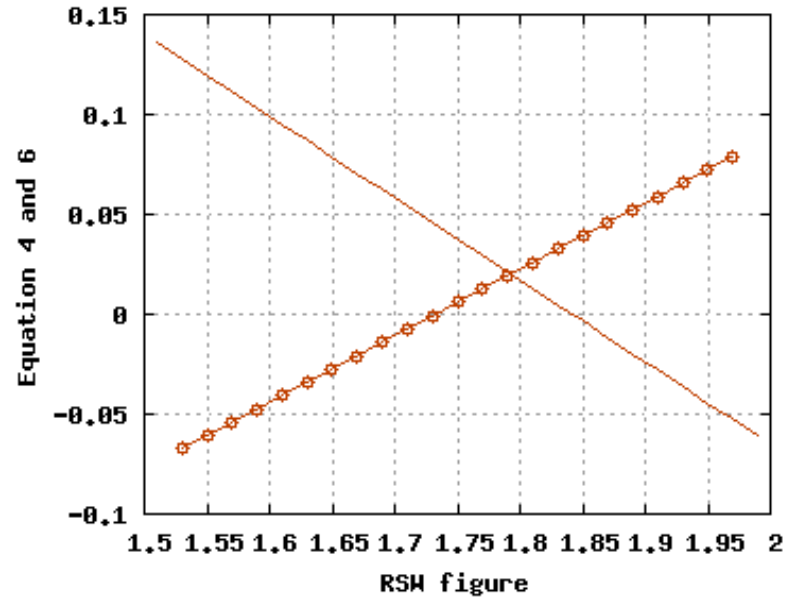

Figure 14: Equation 4 and 6 vs. RSW figure in the vicinity of both the old and the new MPR optimum.

Closely inspecting the curvature in figure 13 just below the original MPR optimum reveals that the new optimum appears to be the point where the curve has its steepest decent towards the original RSW condition. This point can be calculated by determining the second derivative of the original RSW function (equation 4), which is the same as the third derivative of the Bessel function of the first kind and order 1 with respect to its argument. The result is:

$$
\begin{gathered}
J_{1}^{(3)}\left(\beta_{T M_{0}} b\right)=0 \\
\text { or } \\
\beta_{T M_{0}} b=x_{1 m}^{(3)}=1.732
\end{gathered}
$$

The original RSW definition as given in equation $6(\mathrm{o})$ is plotted as a function of the RSW figure 13 and 14 as well. The theoretical MPR optimum RSW figure corresponds very well with the experimentally found value. 


\section{Conclusion}

This paper showed that the original reduced surface wave based multi-path suppression optimum is sub-optimal when it comes to multi-path signals caused by ground reflections.

An improved multi-path suppression optimum was defined based on the MPR definition. A theoretical link between the old and new multi-path suppression optimum is established.

\section{Acknowledgements}

The first author would like to thank Daniël Orban, CEO and founder of Orban Microwave Products, for the ability to pursue the $\mathrm{PhD}$ degree while working at OMP.

\section{References}

[1] L. Boccia, et al., "A dual frequency microstrip patch antenna for high-precision GPS applications", IEEE Antennas and Wireless Propagation Letters, Vol. 3 Is. 1, pp. 157-160, 2004

[2] D.R. Jackson, et al., "Microstrip patch designs that do not excite surface waves", IEEE Trans. Ant. \& Prop., Vol. 41 Is. 8, pp. 1026-1037, 1993

[3] G.J.K. Moernaut \& G.A.E. Vandenbosch, "Simple pen and paper design of shorted annular ring antenna", IEE Electronics Letters, Vol. 39 Is. 25, pp. 1784-1785, 2003

[4] D.M. Pozar, "Microwave engineering ( $2^{\text {nd }}$ edition)", Wiley 